\title{
Sample preparation method for the speciation of polybrominated diphenyl ethers and their methoxylated and hydroxylated analogues in diverse environmental matrices
}

\author{
Jianteng Sun, Jiyan Liu*, Qian Liu, Guangbo Qu, Ting Ruan, Guibin Jiang \\ State Key Laboratory of Environmental Chemistry and Ecotoxicology, Research Center for Eco-Environmental Sciences, Chinese Academy of Sciences, P.O. Box 2871, Beijing 100085, \\ China
}

\section{A R T I C L E I N F O}

\section{Article history:}

Received 7 September 2011

Received in revised form

15 November 2011

Accepted 15 November 2011

Available online 26 November 2011

Keywords:

MeO-PBDEs

OH-PBDES

LC-MS/MS

Sample preparation

Environmental matrices

\begin{abstract}
A B S T R A C T
A reliable analytical method was developed here for the simultaneous separation, identification and quantification of ten polybrominated diphenyl ethers (PBDEs), nine methoxylated PBDEs (MeO-PBDEs) and ten hydroxylated PBDEs (OH-PBDEs) in various environmental matrices, including water, soil, sediment, plant, mollusk and fish. PBDEs and MeO-PBDEs were measured by gas chromatography coupled with mass spectrometry (GC/MS) and liquid chromatography coupled with electrospray ionization (negative)tandem quadrupole mass spectrometry (LC/ESI(-)-MS/MS) for the separation and determination of OH-PBDEs without prior derivatization. After preliminary sample cleaning using acid silica gel, waterimpregnated silica column separation of PBDEs, MeO-PBDEs and OH-PBDEs was proved to be rapid, simple, and efficient. For phenolic analytes, the method detection limits (MDLs) were 3.2-11.6 pg/L in water sample and $2.8-18.4 \mathrm{pg} / \mathrm{g}$ dry weight in solid samples. For neutral compounds, MDLs were $48.8-150.3 \mathrm{pg} / \mathrm{L}$ in water sample and 46.5-170.8 pg/g dry weight in solid samples. The method was validated using six kinds of environmental samples spiked with all analytes at three concentration levels ( $0.3 \mathrm{ng}, 2 \mathrm{ng}$ and $5 \mathrm{ng}$, respectively) for recovery (71-113\%) and repeatability determination (4-12\%RSD).
\end{abstract}

(C) 2011 Elsevier B.V. All rights reserved.

\section{Introduction}

Polybrominated diphenyl ethers (PBDEs) are widely distributed in the environment as flame retardants. As structural analogues to PBDEs, hydroxylated polybrominated diphenyl ethers (OH-PBDEs) and methoxylated polybrominated diphenyl ethers (MeO-PBDEs) have been found in water samples [1], red algae [2] as well as marine animals [3-7], and even in human blood and breast milk $[8,9]$. These compounds are persistent and lipophilic and can be bio-accumulated via the food chain [10]. Both types of analogues present health risks such as thyroid disruptions and neurotoxic effects [11-14]. MeO-PBDEs are often identified as natural products $[15,16]$. However, some reports indicated that MeO-PBDEs may be formed via methylation of PBDEs or OH-PBDEs [4]. OHPBDEs are known of natural origin $[17,18]$ and the products of PBDEs metabolism [19-23]. Recently, Wan et al. [24] found the demethylation of MeO-PBDEs could be a source of OH-PBDEs. The unclear biological process and greater toxicities relative to

\footnotetext{
* Corresponding author. Tel.: +8610 62849334; fax: +86 1062849339 .

E-mail address: liujy@rcees.ac.cn (J. Liu).
}

PBDEs have attracted increasing interests in environmental fate and toxicology of OH-PBDEs and MeO-PBDEs in biota and abiotic environment.

The analysis of PBDEs and MeO-PBDEs is mainly based on GC-MS or GC-ECD [5,25-28]. Although some works used LC-MS/MS methods for the analysis of PBDEs and MeO-PBDEs [29-31], the high quantification limits made it unsuitable for the detection of trace PBDEs and MeO-PBDEs in environmental samples. OH-PBDEs can be determined by GC/MS as their MeOanalogues via a methylated derivatization using diazomethane $[10,25,26,32]$, but the toxicity and easy explosive of diazomethane make the analysis difficult to handle. Therefore, atmospheric pressure chemical ionization (APCI)-LC/MS/MS [29,33] and ionspray ionization (ISP)-LC/MS/MS [34] methods have been developed for the analysis of OH-PBDEs without the time consuming step of derivatization. However, the selectivity and sensitivity of LC-MS/MS methods need to be further improved to make it more efficient in OH-PBDEs analysis. Phenolic compounds were usually separated from neutral compounds by partitioning with potassium hydroxide after extraction. Thus the mentioned methods are always time-consuming and cause the loss of analytes [10,25,26] Kato et al. [29] proposed a single cleanup procedure using gel permeation chromatography (GPC) for simultaneous collection of 
OH-PBDEs and MeO-PBDEs but limited to only some types of matrices such as biosamples.

The aim of this work was to develop a highly sensitive and effective analytical method for simultaneous identification and quantification of ten PBDE congeners, nine MeO-PBDE congeners and ten OH-PBDE congeners in diverse environmental matrices using GC/MS and LC/ESI-MS/MS. A faster and simpler clean-up and pre-separation method for various environmental samples was developed. Water, soil, sediment, plant, mollusk and fish samples were employed to explore the applicability of the method to various environmental matrices. The performance of the overall method was validated in terms of linearity, recovery, repeatability, and sensitivity.

\section{Experimental}

\subsection{Chemicals and reagents}

Nine stock standards of methoxylated polybrominated diphenyl ethers, 4-MeO-BDE-42 $(10 \mu \mathrm{g} / \mathrm{mL}), 4^{\prime}$-MeO-BDE-49 $(10 \mu \mathrm{g} / \mathrm{mL})$, 3-MeO-BDE-47 (50 $\mu \mathrm{g} / \mathrm{mL}), 5-\mathrm{MeO}-\mathrm{BDE}-47$ (50 $\mu \mathrm{g} / \mathrm{mL}), 6-\mathrm{MeO}-$ BDE-47 $(10 \mu \mathrm{g} / \mathrm{mL}), 2^{\prime}-\mathrm{MeO}-\mathrm{BDE}-68(10 \mu \mathrm{g} / \mathrm{mL}), 6-\mathrm{MeO}-\mathrm{BDE}-85$ $(10 \mu \mathrm{g} / \mathrm{mL}), \quad 5^{\prime}-\mathrm{MeO}-\mathrm{BDE}-99 \quad(10 \mu \mathrm{g} / \mathrm{mL}), \quad 6^{\prime}$-MeO-BDE-99 $(10 \mu \mathrm{g} / \mathrm{mL})$, ten hydroxylated polybrominated diphenyl ethers, $3^{\prime}-$ OH-BDE-28 $(50 \mu \mathrm{g} / \mathrm{mL}), 4-\mathrm{OH}-\mathrm{BDE}-42(10 \mu \mathrm{g} / \mathrm{mL}), 4^{\prime}-\mathrm{OH}-\mathrm{BDE}-49$ (10 $\mu \mathrm{g} / \mathrm{mL}), 3-\mathrm{OH}-\mathrm{BDE}-47$ (50 $\mu \mathrm{g} / \mathrm{mL}), 5-\mathrm{OH}-\mathrm{BDE}-47(50 \mu \mathrm{g} / \mathrm{mL})$, 6-OH-BDE-47 $(10 \mu \mathrm{g} / \mathrm{mL}), \quad 2^{\prime}-\mathrm{OH}-\mathrm{BDE}-68 \quad(10 \mu \mathrm{g} / \mathrm{mL}), \quad 6-\mathrm{OH}-$ BDE-85 $(10 \mu \mathrm{g} / \mathrm{mL}), \quad 5^{\prime}-\mathrm{OH}-\mathrm{BDE}-99(10 \mu \mathrm{g} / \mathrm{mL}), \quad 6^{\prime}-\mathrm{OH}-\mathrm{BDE}-99$ $(10 \mu \mathrm{g} / \mathrm{mL})$ and ten standards of polybrominated diphenyl ethers, BDE-28 $(50 \mu \mathrm{g} / \mathrm{mL})$, BDE-47 $(50 \mu \mathrm{g} / \mathrm{mL})$, BDE-66 $(50 \mu \mathrm{g} / \mathrm{mL})$, BDE-68 $(50 \mu \mathrm{g} / \mathrm{mL})$, BDE-85 $(50 \mu \mathrm{g} / \mathrm{mL})$, BDE-99 $(50 \mu \mathrm{g} / \mathrm{mL})$, BDE-138 $(50 \mu \mathrm{g} / \mathrm{mL})$, BDE-153 $(50 \mu \mathrm{g} / \mathrm{mL})$, BDE-154 $(50 \mu \mathrm{g} / \mathrm{mL})$ and BDE-183 $(50 \mu \mathrm{g} / \mathrm{mL})$ were purchased from AccuStandard (New Haven, CT, USA). Recovery surrogate standards, ${ }^{13} \mathrm{C}-66^{\prime}-\mathrm{MeO}-$ BDE100, ${ }^{13} \mathrm{C}-6{ }^{\prime}-\mathrm{OH}-\mathrm{BDE} 100$ and ${ }^{13} \mathrm{C}-\mathrm{BDE}-99$ and injection internal standards, ${ }^{13} \mathrm{C}-6-\mathrm{MeO}-\mathrm{BDE} 47,{ }^{13} \mathrm{C}-6-\mathrm{OH}-\mathrm{BDE} 47$ and ${ }^{13} \mathrm{C}-\mathrm{BDE}-47$ at $50 \mu \mathrm{g} / \mathrm{mL}$ were purchased from Wellington (Guelph, ON, Canada). Individual PBDEs and MeO-PBDEs working solutions were prepared at $500 \mathrm{ng} / \mathrm{mL}$ in hexane and $\mathrm{OH}-\mathrm{PBDEs}$ working solutions were prepared at $500 \mathrm{ng} / \mathrm{mL}$ in acetonitrile.

Methyl tert-butyl ether (MTBE), methanol and acetonitrile were HPLC grade, hexane and dichloromethane (DCM) were pesticide grade and all purchased from J.T. Baker (Phillipsburg, NJ, USA) and Honeywell Burdick \& Jackson (Seelze, Germany). Deionized water $(18.2 \mathrm{M} \Omega$ ) was obtained from ultrapure water purification system (Barnstead International, Dubuque, USA).

Silica gel (0.063-0.100 mm) (Merck, Darmstadt, Germany) and Florisil (0.150-0.250 mm) (Sigma-Aldrich, St Louis, USA) were prebaked at $140^{\circ} \mathrm{C}$ for $7 \mathrm{~h}$. Acid silica was prepared by $56 \mathrm{~g}$ activated silica with $44 \mathrm{~g}$ concentrated $\mathrm{H}_{2} \mathrm{SO}_{4}$. Water-impregnated silica was prepared by $95 \mathrm{~g}$ activated silica with $5 \mathrm{~g}$ deionized water.

\subsection{Sampling and sample preparation}

Six different environmental matrices were selected for spiking experiments to evaluate method performance. Water and sediment samples were collected from Gaobeidian Lake, Beijing in 2010. Surface water samples were collected using $4 \mathrm{~L}$ amber glass bottles and immediately transferred into the laboratory. Sediment samples were collected by a grab sampler. Agricultural soil and leaves of cushaw were obtained in Zhejiang Province, China in 2010. Mollusk and fish samples, Mytilus edulis and Pseudosciaena polyactis were collected from the Bohai Sea and the Donghai Sea, China in 2009, respectively. Mya arenaria was collected from Tianjin, China in 2009 . All the solid samples were stored at $-20^{\circ} \mathrm{C}$ until analysis.

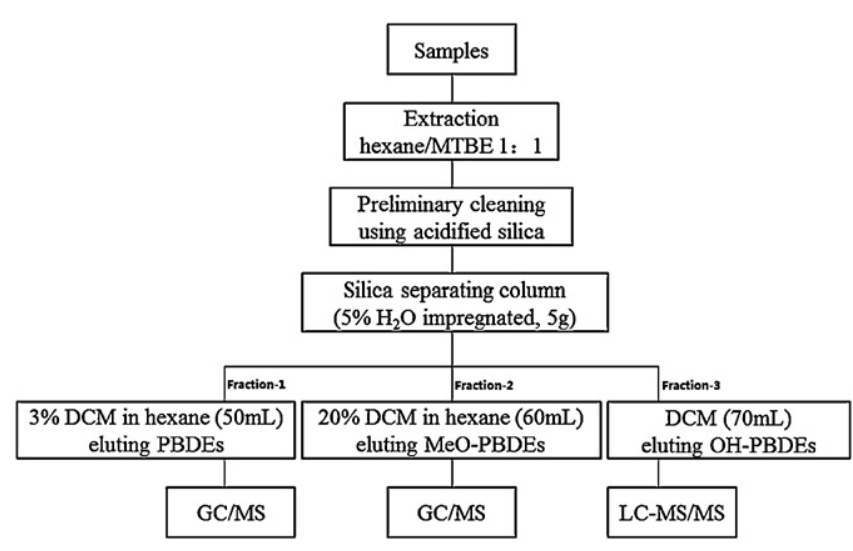

Fig. 1. Optimized extraction and cleanup procedure of environmental samples for the simultaneous separation of PBDEs, MeO-PBDEs and OH-PBDEs.

Before analysis, solid samples were freeze-dried, ground and sieved through a stainless steel 75 -mesh $(0.5 \mathrm{~mm})$ sieve. The mollusk samples were mixtures of the soft tissues from several mussels. $500 \mathrm{~mL}$ lake water and $1.0 \mathrm{~g}$ solid samples were spiked with three recovery surrogate standards ( $5 \mathrm{ng}$ for each) and mixture of ten PBDEs, nine MeO-PBDEs and ten OH-PBDEs (three spiking levels: $0.3 \mathrm{ng}$, $2 \mathrm{ng}$ and $5 \mathrm{ng}$ for each of the analytes). The pretreatment procedure is illustrated in Fig. 1. Lake water sample was extracted twice with $25 \mathrm{~mL}$ hexane/MTBE $(1: 1 ; \mathrm{v} / \mathrm{v})$ after the addition of $2 \mathrm{~mL} 2$ propanol. The extracts were combined and concentrated. The solid samples were extracted with $5 \mathrm{~mL}$ hexane/MTBE $(1: 1 ; \mathrm{v} / \mathrm{v})$ in an ultrasonic bath for $20 \mathrm{~min}$ after the addition of $2 \mathrm{~mL} 2$-propanol. The samples were centrifuged at $3000 \mathrm{rpm}$ for $10 \mathrm{~min}$ and the solvent was transferred to clean vials. This extraction procedure was repeated three times and the extracts were combined and dried under gentle flow of nitrogen gas. The dried residues were then dissolved in $20 \mathrm{~mL}$ of DCM and cleaned by acid silica gel. For sediment sample, sulfur was removed by $2 \mathrm{~g}$ activated copper powder before adding acid silica gel. The acid silica gel was removed through an anhydrous $\mathrm{Na}_{2} \mathrm{SO}_{4}$ column $(4 \mathrm{~g})$. DCM $(40 \mathrm{~mL})$ was used as eluent to ensure all the compounds were desorbed from acid silica gel. The collected extracts were concentrated using a rotary evaporator and re-dissolved with $1 \mathrm{~mL}$ of hexane. Then, the extract was cleaned by a multi-layered column consisting, from bottom to top with $5 \mathrm{~g}$ of silica deactivated with $5 \%$ water $(\mathrm{w} / \mathrm{w})$ and $1 \mathrm{~g}$ of $\mathrm{Na}_{2} \mathrm{SO}_{4}$ for fractionation of PBDEs, MeO-PBDE and OH-PBDEs. After prewashing with $30 \mathrm{~mL}$ of hexane, the extract was loaded to the column. $50 \mathrm{~mL}$ 3\% DCM in hexane was used to elute PBDEs firstly. Secondly, $20 \% \mathrm{DCM}$ in hexane $(60 \mathrm{~mL})$ was applied to elute MeO-PBDEs from the column and finally, DCM $(70 \mathrm{~mL})$ was used to elute OH-PBDEs. Fractions of PBDEs and MeO-PBDEs were concentrated to $100 \mu \mathrm{L}$ and added internal standards $\left({ }^{13} \mathrm{C}-\mathrm{BDE}-47\right.$ and $\left.{ }^{13} \mathrm{C}-6-\mathrm{MeO}-\mathrm{BDE} 47\right)$ prior to instrument analysis. Fraction of $\mathrm{OH}-\mathrm{PBDEs}$ was evaporated to dryness and solvent exchanged with $100 \mu \mathrm{L}$ acetonitrile. ${ }^{13} \mathrm{C}-6$ $\mathrm{OH}-\mathrm{BDE} 47$ was added prior to LC-MS/MS analysis.

\subsection{GC/MS analysis}

PBDEs and MeO-PBDEs analysis was performed on an Agilent Model 6890 gas chromatograph (GC) coupled with a 5973C mass spectrometer (MS) detector (Agilent Technologies, Palo Alto, CA). Samples were injected by a 7683B Series Injector into a DB-5MS column (J \& W Scientific, Folsom, CA, $30 \mathrm{~m}, 0.25 \mathrm{~mm}$ i.d., $0.1 \mu \mathrm{m}$ film thickness) with splitless mode $\left(280^{\circ} \mathrm{C}\right)$. Helium was used as carrier gas at a constant flow of $1.0 \mathrm{~mL} \mathrm{~min}^{-1}$. The oven program started at $90^{\circ} \mathrm{C}$, increased at $15^{\circ} \mathrm{C} \mathrm{min}^{-1}$ to $220^{\circ} \mathrm{C}$, then at $1^{\circ} \mathrm{C} \mathrm{min}^{-1}$ to $260{ }^{\circ} \mathrm{C}$. The post run was set at $300^{\circ} \mathrm{C}$, held for $3 \mathrm{~min}$. The total run 
Table 1

List of target PBDEs and MeO-BDEs and their retention times $\left(t_{\mathrm{R}}\right)$ and SIM ions obtained with GC/MS on DB-5 MS column.

\begin{tabular}{lllll}
\hline Compounds & Formula & GC $t_{\mathrm{R}}(\mathrm{min})$ & \multicolumn{2}{l}{$\mathrm{GC} / \mathrm{MS}$ ions monitored } \\
\cline { 3 - 5 } & & & $m / z 1$ & $m / z 2$ \\
\hline BDE-28 & $\mathrm{C}_{12} \mathrm{H}_{7} \mathrm{OBr}_{3}$ & 10.637 & 245.8 & 247.8 \\
BDE-47 & $\mathrm{C}_{12} \mathrm{H}_{6} \mathrm{OBr}_{4}$ & 13.671 & 325.8 & 327.8 \\
BDE-66 & $\mathrm{C}_{12} \mathrm{H}_{6} \mathrm{OBr}_{4}$ & 14.354 & 325.8 & 327.8 \\
BDE-68 & $\mathrm{C}_{12} \mathrm{H}_{6} \mathrm{OBr}_{4}$ & 13.114 & 325.8 & 327.8 \\
BDE-85 & $\mathrm{C}_{12} \mathrm{H}_{5} \mathrm{OBr}_{5}$ & 21.660 & 405.8 & 407.8 \\
BDE-99 & $\mathrm{C}_{12} \mathrm{H}_{5} \mathrm{OBr}_{5}$ & 18.817 & 405.8 & 407.8 \\
BDE-138 & $\mathrm{C}_{12} \mathrm{H}_{4} \mathrm{OBr}_{6}$ & 30.514 & 485.8 & 487.8 \\
BDE-153 & $\mathrm{C}_{12} \mathrm{H}_{4} \mathrm{OBr}_{6}$ & 26.413 & 485.8 & 487.8 \\
BDE-154 & $\mathrm{C}_{12} \mathrm{H}_{4} \mathrm{OBr}_{6}$ & 23.327 & 485.8 & 487.8 \\
BDE-183 & $\mathrm{C}_{12} \mathrm{H}_{3} \mathrm{OBr}_{7}$ & 35.955 & 565.8 & 567.8 \\
2'-MeO-BDE68 & $\mathrm{C}_{13} \mathrm{H}_{8} \mathrm{O}_{2} \mathrm{Br}_{4}$ & 15.483 & 515.7 & 517.8 \\
6-MeO-BDE47 & $\mathrm{C}_{13} \mathrm{H}_{8} \mathrm{O}_{2} \mathrm{Br}_{4}$ & 16.139 & 515.7 & 517.8 \\
3-MeO-BDE47 & $\mathrm{C}_{13} \mathrm{H}_{8} \mathrm{O}_{2} \mathrm{Br}_{4}$ & 17.143 & 515.7 & 517.8 \\
5-MeO-BDE47 & $\mathrm{C}_{13} \mathrm{H}_{8} \mathrm{O}_{2} \mathrm{Br}_{4}$ & 17.534 & 515.7 & 517.8 \\
4'-MeO-BDE49 & $\mathrm{C}_{13} \mathrm{H}_{8} \mathrm{O}_{2} \mathrm{Br}_{4}$ & 17.855 & 515.7 & 517.8 \\
4-MeO-BDE42 & $\mathrm{C}_{13} \mathrm{H}_{8} \mathrm{O}_{2} \mathrm{Br}_{4}$ & 20.421 & 515.7 & 517.8 \\
6'-MeO-BDE99 & $\mathrm{C}_{13} \mathrm{H}_{7} \mathrm{O}_{2} \mathrm{Br}_{5}$ & 21.902 & 595.7 & 597.8 \\
5'-MeO-BDE99 & $\mathrm{C}_{13} \mathrm{H}_{7} \mathrm{O}_{2} \mathrm{Br}_{5}$ & 24.360 & 595.7 & 597.8 \\
6-MeO-BDE85 & $\mathrm{C}_{13} \mathrm{H}_{7} \mathrm{O}_{2} \mathrm{Br}_{5}$ & 25.715 & 595.7 & 597.8 \\
\hline
\end{tabular}

time was 51.67 min. Quantitative determination by GC-MS (EI) was in the selected ion monitoring (SIM) mode. Quantification ions were $[\mathrm{M}]^{+}$or $[\mathrm{M}-2 \mathrm{Br}]^{+}$and identity confirmation ions were $[\mathrm{M}+2]^{+}$or $[(\mathrm{M}+2)-2 \mathrm{Br}]^{+}$. The ions monitored for PBDEs and MeO-PBDEs are presented in Table 1.

\subsection{LC-MS/MS analysis}

Analysis of OH-PBDEs was carried out using an Agilent 1290 Series LC system coupled with an Agilent 6460 Triple Quadrupole MS/MS system (Agilent Technologies, Palo Alto, CA) using a C18 column $(100 \mathrm{~mm} \times 2.1 \mathrm{~mm}, 2.2 \mu \mathrm{m}$ particle size, Dionex, USA). The mobile phase consisting of acetonitrile (Solvent A) and water (Solvent $B$ ) was used with a gradient elution of A:B from 55:45 to 75:25 in $20 \mathrm{~min}$ at a flow rate of $0.38 \mathrm{~mL} \mathrm{~min}^{-1}$. The column was equilibrated for $5 \mathrm{~min}$ between runs. The column temperature was set at $40^{\circ} \mathrm{C}$ and the volume injected onto the column was $10 \mu \mathrm{L}$.

Mass spectrometric detection was completed using ESI source in the negative ion multiple-reaction monitoring (MRM) mode. An Agilent Jet Stream was coupled to ESI source. Nitrogen was used as the curtain, nebulizer gas and collision gas. The flow was optimized at $5 \mathrm{~L} / \mathrm{min}$ at a constant temperature of $350^{\circ} \mathrm{C}$ and the sheath gas flow was optimized at $11 \mathrm{~L} / \mathrm{min}$ at a constant temperature of $400^{\circ} \mathrm{C}$. The capillary and nozzle voltages were held at $3500 \mathrm{~V}$ and $500 \mathrm{~V}$, respectively. Nebulizer pressure and Delta electron multiplier voltage (EMV) was set as $50 \mathrm{psi}$ and $800 \mathrm{~V}$, respectively. A dwell time of $20 \mathrm{~ms}$ was used for each of the MRM transitions. The formula, LC retention time, and the MRM transitions of all analyzed OH-PBDEs are summarized in Table 2.

\subsection{Quality assurance and quality control}

Seven-point standard calibration curves were prepared using standards with concentration of 2, 5, 10, 25, 50, 100 and $200 \mathrm{ng} / \mathrm{mL}$ and surrogate and internal standards at constant concentration of $50 \mathrm{ng} / \mathrm{mL}$. The equipment detection limits (EDLs) for all the investigated compounds by GC/MS and LC-MS/MS were estimated based on a signal-to-noise ratio $(\mathrm{S} / \mathrm{N})$ of 3 using the lowest concentration standard. The same criteria were used to determine the method detection limits (MDLs) from spiked matrices. Repeatability was evaluated by intra- and inter-assay variation. Intra-assay variation was assessed by five consecutive injections of a blank soil sample spiked at $5 \mathrm{ng}$, and inter-assay variation was determined by measuring the same spiking sample each day for five consecutive days. All targets could be identified by retention time at each characteristic ion. The calibration of working curves was done daily to monitor possible carryover effect between runs and adjust retention time variations. No memory effects were observed between consecutive runs of the spiking concentration, and the target compounds were under MDL in solvents.

\section{Results and discussion}

\subsection{Optimization of sample preparation conditions}

The formula and GC retention time of target PBDEs and MeOPBDEs are listed in Table 1.

Sample preparation was designed to fractionate PBDEs, MeOPBDEs and $\mathrm{OH}-\mathrm{PBDEs}$ within a single separation column. In our experiment, different clean-up procedures were explored. Several chromatographic columns were tested for clean-up procedure including Florisil, acid silica gel and neutral silica gel. The proportions of hexane and DCM were also optimized as eluent. The results showed co-elution existed among MeO-PBDE congeners and OH-PBDE congeners on acid silica gel column no matter how much $\mathrm{H}_{2} \mathrm{SO}_{4}$ was used to acidify the silica gel. The recovery of $3-\mathrm{MeO}-\mathrm{BDE}-47$ was only $27 \%$, and co-eluted by $3-\mathrm{OH}-\mathrm{BDE}-47$ (recovery 25\%) and 5-OH-BDE-47 (recovery 31\%) when using $80 \%$ hexane/DCM as eluent. For Florisil column, PBDEs and MeO-PBDEs could be eluted separately while OH-PBDEs were strongly adsorbed even though Florisil was deactivated by water (1.2-5\%). Almost all the OH-PBDEs had low recoveries (<50\%) except 3'-OH-BDE28 ( $90 \%)$. Activated neutral silica showed similar performance to Florisil when using DCM as eluent. However, good separating property and eluting capability could be obtained when silica was impregnated with $5 \%$ water. Finally, 5\% water impregnated silica column was chosen as separating column and the optimized loading amount was $5 \mathrm{~g}$. The mixture of hexane and DCM was optimized from $100 \%$ hexane to $100 \%$ DCM and the eluent was established as $50 \mathrm{~mL}$ of $3 \% \mathrm{DCM} /$ hexane for PBDEs, $60 \mathrm{~mL}$ of $20 \%$ $\mathrm{DCM} /$ hexane for MeO-PBDEs and $70 \mathrm{~mL}$ of DCM for OH-PBDEs. In order to remove abundant impurities in the environmental samples, GPC and acid silica gel $\left(44 \% \mathrm{H}_{2} \mathrm{SO}_{4}\right.$ acidified) were tested for the preliminary purification procedure. Although both methods showed good clean-up performance, acid silica gel was chosen finally because less solvent and less time was needed. For sediment sample, activated copper powder was used for elimination of sulfur-containing compounds. Although all target PBDEs and MeOPBDEs can be separated by a DB-5 MS column and determined simultaneously by GC/MS, the fractionation of PBDEs and MeOPBDEs on water-impregnated silica column could decrease the interferences when more PBDEs and MeO-PBDEs species were analyzed. In addition, solid-phase extraction (SPE) method was tried and C18 and HLB columns (Waters, Canada) were tested. Although less solvent was needed by SPE method, lower recoveries and worse separation effect were obtained.

Comparing with the methods reported in the previous papers $[25,26]$ in which partitioning with potassium hydroxide and subsequent acidification and back extraction of the aqueous fraction was always used to separate the neutral and phenolic analogues, a single $5 \%$ water impregnated silica column was used instead in this paper for the simultaneous separation of three analogues, PBDEs, MeO-PBDEs and OH-PBDEs. Derivatization of OH-PBDEs was unnecessary so that the entire preparation was simplified.

\subsection{Optimization of LC-MS/MS conditions}

Five HPLC chromatographic columns were tested to resolve the coelution of isomeric species, including ZORBAX C18 column 
Table 2

Characterization of target OH-PBDEs and MRM parameters used for qualitative and quantitative determination.

\begin{tabular}{|c|c|c|c|c|c|c|c|}
\hline Compounds & Formula & $\mathrm{LC} t_{\mathrm{R}}(\min )$ & {$[\mathrm{M}-\mathrm{H}]^{-}$} & Product ions & MRM transition $(\mathrm{m} / \mathrm{z})$ & Frag (V) & $\mathrm{CE}(\mathrm{V})$ \\
\hline 3'-OH-BDE28 & $\mathrm{C}_{12} \mathrm{H}_{7} \mathrm{Br}_{3} \mathrm{O}_{2}$ & 8.058 & 420.7 & $\begin{array}{l}{\left[\mathrm{C}_{12} \mathrm{H}_{6} \mathrm{Br}_{2} \mathrm{O}_{2}\right]^{-}} \\
{\left[\mathrm{C}_{6} \mathrm{H}_{3} \mathrm{Br}_{2} \mathrm{O}\right]^{-}} \\
{[\mathrm{Br}]^{-}}\end{array}$ & $\begin{array}{l}420.7 \rightarrow 340.6^{\mathrm{a}} \\
420.7 \rightarrow 80.8^{\mathrm{b}}\end{array}$ & $\begin{array}{l}-160 \\
-160\end{array}$ & $\begin{array}{l}-15 \\
-32\end{array}$ \\
\hline 4-OH-BDE42 & $\mathrm{C}_{12} \mathrm{H}_{6} \mathrm{Br}_{4} \mathrm{O}_{2}$ & 9.669 & 500.7 & $\begin{array}{l}{\left[\mathrm{C}_{6} \mathrm{H}_{2} \mathrm{Br}_{2} \mathrm{O}_{2}\right]^{-}} \\
{[\mathrm{Br}]^{-}}\end{array}$ & $\begin{array}{l}500.7 \rightarrow 265.6^{\mathrm{a}} \\
500.7 \rightarrow 80.8^{\mathrm{b}}\end{array}$ & $\begin{array}{l}-135 \\
-135\end{array}$ & $\begin{array}{l}-22 \\
-40\end{array}$ \\
\hline 3-OH-BDE47 & $\mathrm{C}_{12} \mathrm{H}_{6} \mathrm{Br}_{4} \mathrm{O}_{2}$ & 10.445 & 500.7 & $\begin{array}{l}{\left[\mathrm{C}_{12} \mathrm{H}_{5} \mathrm{Br}_{3} \mathrm{O}_{2}\right]^{-}} \\
{\left[\mathrm{C}_{12} \mathrm{H}_{5} \mathrm{Br}_{2} \mathrm{O}_{2}\right]^{-}} \\
{\left[\mathrm{C}_{6} \mathrm{H}_{3} \mathrm{Br}_{2} \mathrm{O}\right]^{-}} \\
{[\mathrm{Br}]^{-}}\end{array}$ & $\begin{array}{l}500.7 \rightarrow 418.6^{\mathrm{a}} \\
500.7 \rightarrow 80.8^{\mathrm{b}}\end{array}$ & $\begin{array}{l}-135 \\
-135\end{array}$ & $\begin{array}{l}-38 \\
-40\end{array}$ \\
\hline $4^{\prime}-\mathrm{OH}-\mathrm{BDE} 49$ & $\mathrm{C}_{12} \mathrm{H}_{6} \mathrm{Br}_{4} \mathrm{O}_{2}$ & 10.966 & 500.7 & $\begin{array}{l}{\left[\mathrm{C}_{6} \mathrm{H}_{2} \mathrm{Br}_{2} \mathrm{O}_{2}\right]^{-}} \\
{[\mathrm{Br}]^{-}}\end{array}$ & $\begin{array}{l}500.7 \rightarrow 265.6^{\mathrm{a}} \\
500.7 \rightarrow 80.8^{\mathrm{b}}\end{array}$ & $\begin{array}{l}-135 \\
-135\end{array}$ & $\begin{array}{l}-25 \\
-40\end{array}$ \\
\hline 5-OH-BDE47 & $\mathrm{C}_{12} \mathrm{H}_{6} \mathrm{Br}_{4} \mathrm{O}_{2}$ & 11.207 & 500.7 & $\begin{array}{l}{\left[\mathrm{C}_{12} \mathrm{H}_{5} \mathrm{Br}_{3} \mathrm{O}_{2}\right]^{-}} \\
{\left[\mathrm{C}_{12} \mathrm{H}_{5} \mathrm{Br}_{2} \mathrm{O}_{2}\right]^{-}} \\
{\left[\mathrm{C}_{6} \mathrm{H}_{3} \mathrm{Br}_{2} \mathrm{O}\right]^{-}} \\
{[\mathrm{Br}]^{-}}\end{array}$ & $\begin{array}{l}500.7 \rightarrow 418.6^{\mathrm{a}} \\
500.7 \rightarrow 80.8^{\mathrm{b}}\end{array}$ & $\begin{array}{l}-100 \\
-100\end{array}$ & $\begin{array}{l}-20 \\
-32\end{array}$ \\
\hline 6-OH-BDE47 & $\mathrm{C}_{12} \mathrm{H}_{6} \mathrm{Br}_{4} \mathrm{O}_{2}$ & 12.002 & 500.7 & {$[\mathrm{Br}]^{-}$} & $\begin{array}{l}500.7 \rightarrow 80.8^{a} \\
500.7 \rightarrow 78.7^{b}\end{array}$ & $\begin{array}{l}-100 \\
-100\end{array}$ & $\begin{array}{r}-8 \\
-30\end{array}$ \\
\hline 2'-OH-BDE68 & $\mathrm{C}_{12} \mathrm{H}_{6} \mathrm{Br}_{4} \mathrm{O}_{2}$ & 12.838 & 500.7 & {$[\mathrm{Br}]^{-}$} & $\begin{array}{l}500.7 \rightarrow 80.8^{\mathrm{a}} \\
500.7 \rightarrow 78.7^{\mathrm{b}}\end{array}$ & $\begin{array}{l}-100 \\
-100\end{array}$ & $\begin{array}{r}-8 \\
-30\end{array}$ \\
\hline 6-OH-BDE85 & $\mathrm{C}_{12} \mathrm{H}_{5} \mathrm{Br}_{5} \mathrm{O}_{2}$ & 14.158 & 578.6 & {$[\mathrm{Br}]^{-}$} & $\begin{array}{l}578.6 \rightarrow 80.8^{\mathrm{a}} \\
578.6 \rightarrow 78.7^{\mathrm{b}}\end{array}$ & $\begin{array}{l}-100 \\
-100\end{array}$ & $\begin{array}{l}-12 \\
-27\end{array}$ \\
\hline 5'-OH-BDE99 & $\mathrm{C}_{12} \mathrm{H}_{5} \mathrm{Br}_{5} \mathrm{O}_{2}$ & 15.258 & 578.6 & $\begin{array}{l}{\left[\mathrm{C}_{12} \mathrm{H}_{4} \mathrm{Br}_{4} \mathrm{O}_{2}\right]^{-}} \\
{[\mathrm{Br}]^{-}}\end{array}$ & $\begin{array}{l}578.6 \rightarrow 498.2^{\mathrm{a}} \\
578.6 \rightarrow 78.7^{\mathrm{b}}\end{array}$ & $\begin{array}{l}-135 \\
-135\end{array}$ & $\begin{array}{l}-20 \\
-42\end{array}$ \\
\hline 6'-OH-BDE99 & $\mathrm{C}_{12} \mathrm{H}_{5} \mathrm{Br}_{5} \mathrm{O}_{2}$ & 16.166 & 578.6 & {$[\mathrm{Br}]^{-}$} & $\begin{array}{l}578.6 \rightarrow 80.8^{\mathrm{a}} \\
578.6 \rightarrow 78.7^{\mathrm{b}}\end{array}$ & $\begin{array}{l}-120 \\
-120\end{array}$ & $\begin{array}{l}-35 \\
-25\end{array}$ \\
\hline
\end{tabular}

a MRM transition used for quantification.

b MRM transition used for qualitative analysis.

$(150 \mathrm{~mm} \times 3 \mathrm{~mm}, 5 \mu \mathrm{m})$ (Agilent), Acclaim $120 \mathrm{C} 18$ column $(150 \mathrm{~mm} \times 4.6 \mathrm{~mm}, 5 \mu \mathrm{m})$ (Dionex), Acclaim $120 \mathrm{C} 18$ column $(250 \mathrm{~mm} \times 4.6 \mathrm{~mm}, 5 \mu \mathrm{m})$ (Dionex), Acclaim RSLC $120 \mathrm{C} 18 \mathrm{col}-$ umn $(50 \mathrm{~mm} \times 2.1 \mathrm{~mm}, 2.2 \mu \mathrm{m})$ (Dionex) and Acclaim RSLC 120 C18 column $(100 \mathrm{~mm} \times 2.1 \mathrm{~mm}, 2.2 \mu \mathrm{m})$ (Dionex). Among the tested columns, the Dionex Acclaim RSLC 120 C18 column $(100 \mathrm{~mm} \times 2.1 \mathrm{~mm}, 2.2 \mu \mathrm{m})$ presented the best performance for the separation of 10 OH-PBDEs. Fig. 2 shows the total ion chromatogram (TIC) and the individual MRM chromatograms of ten
OH-PBDEs. Different mobile phase, as well as various gradient slopes was also considered. Comparing to the mixture of methanol and water, the mixture of acetonitrile and water are more appropriate to be used as mobile phase to solve the coelution of 6-OH-BDE47 and 2 '-OH-BDE68. Since acetonitrile tended to carbonize the corona pin of APCI ion source and reduce the sensitivity consequently, ESI in negative ion mode was selected as the most suitable ionization. The installation of Agilent Jet Stream can help the ionization of analytes by sheath gas and hence increased the sensitivity.

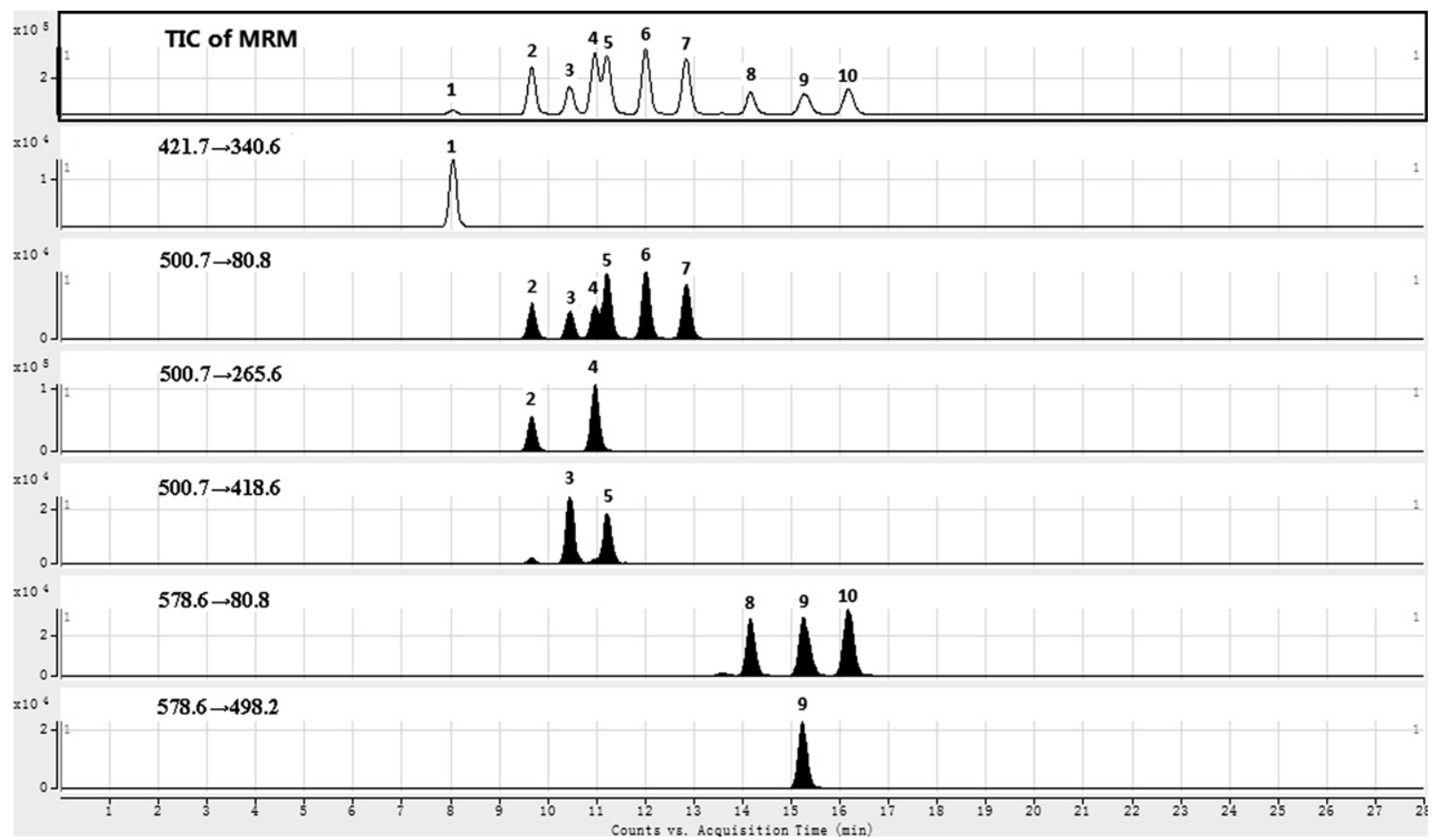

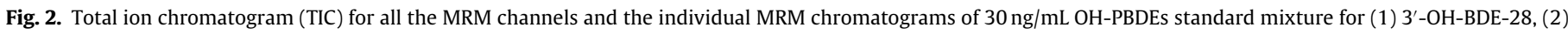
4-OH-BDE-42, (3) 3-OH-BDE-47, (4) 4'-OH-BDE-49, (5) 5-OH-BDE-47, (6) 6-OH-BDE-47, (7) 2'-OH-BDE-68, (8) 6-OH-BDE-85, (9) 5'-OH-BDE-99 and (10) 6'-OH-BDE-99. 
Table 3

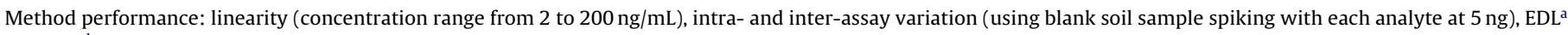
and $\mathrm{MDL}^{\mathrm{b}}(\mathrm{S} / \mathrm{N}=3)$ of all the target analytes for six environmental matrices.

\begin{tabular}{|c|c|c|c|c|c|c|c|c|c|c|}
\hline \multirow[t]{2}{*}{ Compounds } & \multirow[t]{2}{*}{$r^{2 c}$} & \multirow{2}{*}{$\begin{array}{l}\text { Intra-assay } \\
\text { variation } \\
(\% \text { RSD, } n=5)\end{array}$} & \multirow{2}{*}{$\begin{array}{l}\text { Inter-assay } \\
\text { variation } \\
(\% \text { RSD, } n=5)\end{array}$} & \multirow[t]{2}{*}{ EDLs (ng/mL) } & \multicolumn{6}{|l|}{ MDLs } \\
\hline & & & & & $\begin{array}{l}\text { Water } \\
(\mathrm{pg} / \mathrm{L})\end{array}$ & $\begin{array}{l}\text { Soil } \\
\text { (pg/g dw) }\end{array}$ & $\begin{array}{l}\text { Sediment } \\
(\mathrm{pg} / \mathrm{g} \mathrm{dw})\end{array}$ & $\begin{array}{l}\text { Plant } \\
\text { (pg/g dw) }\end{array}$ & $\begin{array}{l}\text { Mollusk } \\
\text { (pg/g dw) }\end{array}$ & $\begin{array}{l}\text { Fish } \\
(\mathrm{pg} / \mathrm{g} \mathrm{dw})\end{array}$ \\
\hline BDE-28 & 0.9994 & 7 & 9 & 0.27 & 53.5 & 56.8 & 68.7 & 55.6 & 69.3 & 70.7 \\
\hline BDE-47 & 0.9995 & 6 & 7 & 0.24 & 48.8 & 46.5 & 57.3 & 49.5 & 56.4 & 59.2 \\
\hline BDE-66 & 0.9991 & 6 & 6 & 0.25 & 61.2 & 55.2 & 62.4 & 67.9 & 69.2 & 66.5 \\
\hline BDE-68 & 0.9992 & 5 & 6 & 0.28 & 80.2 & 68.4 & 78.4 & 54.8 & 67.9 & 78.3 \\
\hline BDE-85 & 0.9984 & 8 & 8 & 0.46 & 64.5 & 85.5 & 80.8 & 76.4 & 66 & 78.4 \\
\hline BDE-99 & 0.9982 & 8 & 9 & 0.41 & 70.4 & 78.8 & 69.4 & 68.4 & 90.5 & 84.6 \\
\hline BDE-138 & 0.9965 & 7 & 6 & 0.48 & 85.5 & 80.9 & 93.8 & 84.5 & 85.2 & 99.2 \\
\hline BDE-153 & 0.9935 & 6 & 10 & 0.56 & 110.4 & 90.2 & 123.5 & 95.2 & 104.7 & 104.8 \\
\hline BDE-154 & 0.9938 & 7 & 8 & 0.52 & 124.5 & 93.7 & 130.2 & 113.4 & 120.6 & 137.1 \\
\hline BDE-183 & 0.9923 & 9 & 11 & 0.75 & 150.3 & 143.5 & 170.8 & 152.7 & 149.2 & 163.6 \\
\hline 2'-MeO-BDE68 & 0.9996 & 5 & 7 & 0.32 & 67.4 & 63.5 & 64.9 & 53.2 & 78.3 & 69.5 \\
\hline 6-MeO-BDE47 & 0.9994 & 5 & 7 & 0.28 & 55.1 & 50.7 & 58.7 & 66.3 & 70.5 & 84.5 \\
\hline 3-MeO-BDE47 & 0.9997 & 7 & 6 & 0.35 & 65.2 & 67.4 & 70.7 & 74.2 & 66.8 & 62.6 \\
\hline 5-MeO-BDE47 & 0.9994 & 6 & 10 & 0.39 & 74.3 & 70.7 & 88.2 & 83.2 & 67.3 & 75.5 \\
\hline $4^{\prime}-\mathrm{MeO}-\mathrm{BDE} 49$ & 0.9992 & 4 & 6 & 0.43 & 75.6 & 74.8 & 79.8 & 84.3 & 87.4 & 88.3 \\
\hline 4-MeO-BDE42 & 0.999 & 7 & 9 & 0.35 & 69.3 & 65.5 & 78.3 & 73.6 & 59.6 & 68.5 \\
\hline 6'-MeO-BDE99 & 0.9981 & 6 & 8 & 0.48 & 90.4 & 85.4 & 105.3 & 96.4 & 83.2 & 96.4 \\
\hline 5'-MeO-BDE99 & 0.998 & 8 & 12 & 0.44 & 87.2 & 82.4 & 88.6 & 99.3 & 75.7 & 82.5 \\
\hline 6-MeO-BDE85 & 0.9958 & 6 & 9 & 0.46 & 83.6 & 94.3 & 96.5 & 89.5 & 81.3 & 90.4 \\
\hline 3'-OH-BDE28 & 0.9922 & 10 & 12 & 0.1 & 11.6 & 10.4 & 18.4 & 11.3 & 12.7 & 14.4 \\
\hline 4-OH-BDE42 & 0.9934 & 7 & 9 & 0.04 & 7.2 & 6.2 & 11.7 & 6.9 & 7.7 & 8.3 \\
\hline 3-OH-BDE47 & 0.9931 & 4 & 5 & 0.05 & 5.5 & 4.3 & 12.2 & 5.5 & 6.8 & 7.5 \\
\hline 4'-OH-BDE49 & 0.998 & 5 & 8 & 0.02 & 4.9 & 3.7 & 5.6 & 7.6 & 5.8 & 6.2 \\
\hline 5-OH-BDE47 & 0.9962 & 5 & 6 & 0.01 & 3.2 & 2.8 & 3.8 & 5.1 & 4.4 & 4.9 \\
\hline 6-OH-BDE47 & 0.9964 & 7 & 9 & 0.03 & 3.8 & 4.9 & 6.8 & 4.2 & 5.6 & 5.7 \\
\hline 2'-OH-BDE68 & 0.9926 & 6 & 8 & 0.04 & 7.8 & 5.3 & 7.1 & 4.6 & 6.9 & 9.5 \\
\hline 6-OH-BDE85 & 0.9947 & 7 & 6 & 0.05 & 8.2 & 4.5 & 5.9 & 9.4 & 6.7 & 5.5 \\
\hline 5'-OH-BDE99 & 0.9951 & 9 & 9 & 0.05 & 4.3 & 8.5 & 6.5 & 7.9 & 9.3 & 6.1 \\
\hline 6'-OH-BDE99 & 0.9963 & 8 & 10 & 0.04 & 6.9 & 7.7 & 8.8 & 6.5 & 9.7 & 10.8 \\
\hline
\end{tabular}

Table 4

Spike recoveries and standard deviation ( $\% \pm \mathrm{SD}, n=3)$ at $0.3,2$ and $5 \mathrm{ng}$ for each target analyte using three different environmental matrices (water, soil, sediment).

\begin{tabular}{|c|c|c|c|c|c|c|c|c|c|}
\hline \multirow[t]{2}{*}{ Compounds } & \multicolumn{3}{|l|}{$\begin{array}{l}\text { Water } \\
\% R \pm \mathrm{SD}\end{array}$} & \multicolumn{3}{|l|}{$\begin{array}{l}\text { Soil } \\
\% R \pm \mathrm{SD}\end{array}$} & \multicolumn{3}{|l|}{$\begin{array}{l}\text { Sediment } \\
\% R \pm \mathrm{SD}\end{array}$} \\
\hline & $3 \mathrm{ng} / \mathrm{mL}$ & $20 \mathrm{ng} / \mathrm{mL}$ & $50 \mathrm{ng} / \mathrm{mL}$ & $3 \mathrm{ng} / \mathrm{mL}$ & $20 \mathrm{ng} / \mathrm{mL}$ & $50 \mathrm{ng} / \mathrm{mL}$ & $3 \mathrm{ng} / \mathrm{mL}$ & $20 \mathrm{ng} / \mathrm{mL}$ & $50 \mathrm{ng} / \mathrm{mL}$ \\
\hline BDE-28 & $81 \pm 7$ & $85 \pm 5$ & $89 \pm 2$ & $83 \pm 10$ & $92 \pm 12$ & $90 \pm 8$ & $76 \pm 8$ & $80 \pm 9$ & $84 \pm 7$ \\
\hline BDE-47 & $79 \pm 8$ & $84 \pm 7$ & $93 \pm 5$ & $78 \pm 8$ & $91 \pm 6$ & $95 \pm 6$ & $81 \pm 13$ & $91 \pm 6$ & $91 \pm 2$ \\
\hline BDE-66 & $83 \pm 5$ & $92 \pm 2$ & $94 \pm 2$ & $85 \pm 6$ & $89 \pm 7$ & $89 \pm 5$ & $83 \pm 6$ & $88 \pm 8$ & $86 \pm 4$ \\
\hline BDE-68 & $78 \pm 5$ & $104 \pm 7$ & $94 \pm 6$ & $76 \pm 5$ & $90 \pm 8$ & $92 \pm 5$ & $74 \pm 9$ & $109 \pm 7$ & $87 \pm 4$ \\
\hline BDE-85 & $84 \pm 8$ & $89 \pm 6$ & $93 \pm 8$ & $81 \pm 8$ & $103 \pm 7$ & $97 \pm 5$ & $73 \pm 10$ & $83 \pm 5$ & $86 \pm 5$ \\
\hline BDE-99 & $80 \pm 7$ & $95 \pm 8$ & $92 \pm 4$ & $83 \pm 7$ & $85 \pm 9$ & $89 \pm 5$ & $80 \pm 8$ & $90 \pm 8$ & $94 \pm 5$ \\
\hline BDE-138 & $85 \pm 9$ & $91 \pm 5$ & $95 \pm 5$ & $91 \pm 14$ & $96 \pm 4$ & $109 \pm 6$ & $82 \pm 12$ & $92 \pm 8$ & $95 \pm 7$ \\
\hline BDE-153 & $82 \pm 8$ & $93 \pm 4$ & $88 \pm 5$ & $90 \pm 5$ & $94 \pm 8$ & $91 \pm 8$ & $113 \pm 9$ & $87 \pm 5$ & $84 \pm 6$ \\
\hline BDE-154 & $111 \pm 6$ & $87 \pm 5$ & $85 \pm 7$ & $81 \pm 9$ & $90 \pm 9$ & $95 \pm 3$ & $82 \pm 7$ & $83 \pm 7$ & $87 \pm 3$ \\
\hline BDE-183 & $80 \pm 5$ & $84 \pm 7$ & $85 \pm 6$ & $80 \pm 9$ & $86 \pm 8$ & $89 \pm 6$ & $83 \pm 14$ & $87 \pm 6$ & $93 \pm 5$ \\
\hline 2'-MeO-BDE68 & $77 \pm 6$ & $93 \pm 4$ & $90 \pm 4$ & $90 \pm 6$ & $94 \pm 5$ & $93 \pm 8$ & $74 \pm 8$ & $88 \pm 8$ & $87 \pm 2$ \\
\hline 6-MeO-BDE47 & $90 \pm 8$ & $91 \pm 6$ & $96 \pm 5$ & $90 \pm 6$ & $92 \pm 7$ & $95 \pm 8$ & $76 \pm 11$ & $94 \pm 12$ & $92 \pm 7$ \\
\hline 3-MeO-BDE47 & $84 \pm 12$ & $93 \pm 7$ & $91 \pm 8$ & $73 \pm 13$ & $95 \pm 7$ & $91 \pm 7$ & $83 \pm 5$ & $91 \pm 5$ & $94 \pm 3$ \\
\hline 5-MeO-BDE47 & $85 \pm 9$ & $88 \pm 6$ & $94 \pm 4$ & $108 \pm 6$ & $92 \pm 8$ & $97 \pm 6$ & $84 \pm 9$ & $90 \pm 7$ & $93 \pm 7$ \\
\hline 4'-MeO-BDE49 & $86 \pm 4$ & $89 \pm 11$ & $96 \pm 3$ & $79 \pm 15$ & $91 \pm 7$ & $96 \pm 2$ & $80 \pm 9$ & $86 \pm 9$ & $108 \pm 7$ \\
\hline 4-MeO-BDE42 & $81 \pm 12$ & $84 \pm 6$ & $92 \pm 6$ & $85 \pm 9$ & $90 \pm 6$ & $93 \pm 3$ & $85 \pm 13$ & $87 \pm 8$ & $89 \pm 6$ \\
\hline 6'-MeO-BDE99 & $85 \pm 6$ & $90 \pm 6$ & $93 \pm 4$ & $76 \pm 12$ & $88 \pm 8$ & $95 \pm 8$ & $91 \pm 9$ & $91 \pm 7$ & $96 \pm 3$ \\
\hline 5'-MeO-BDE99 & $77 \pm 8$ & $93 \pm 9$ & $95 \pm 6$ & $83 \pm 7$ & $87 \pm 4$ & $97 \pm 3$ & $77 \pm 10$ & $90 \pm 16$ & $94 \pm 6$ \\
\hline 6-MeO-BDE85 & $84 \pm 9$ & $91 \pm 13$ & $97 \pm 6$ & $87 \pm 9$ & $85 \pm 7$ & $91 \pm 7$ & $82 \pm 9$ & $87 \pm 9$ & $86 \pm 12$ \\
\hline 3'-OH-BDE28 & $74 \pm 6$ & $84 \pm 7$ & $77 \pm 8$ & $84 \pm 8$ & $86 \pm 8$ & $89 \pm 12$ & $71 \pm 7$ & $74 \pm 12$ & $78 \pm 10$ \\
\hline 4-OH-BDE42 & $85 \pm 13$ & $89 \pm 8$ & $87 \pm 8$ & $87 \pm 5$ & $87 \pm 14$ & $85 \pm 8$ & $76 \pm 6$ & $79 \pm 3$ & $77 \pm 9$ \\
\hline 3-OH-BDE47 & $86 \pm 7$ & $92 \pm 7$ & $104 \pm 4$ & $77 \pm 8$ & $97 \pm 8$ & $93 \pm 10$ & $86 \pm 13$ & $90 \pm 10$ & $91 \pm 5$ \\
\hline $4^{\prime}-\mathrm{OH}-\mathrm{BDE} 49$ & $86 \pm 6$ & $86 \pm 9$ & $92 \pm 5$ & $85 \pm 8$ & $82 \pm 5$ & $88 \pm 5$ & $76 \pm 8$ & $83 \pm 7$ & $86 \pm 4$ \\
\hline 5-OH-BDE47 & $79 \pm 15$ & $91 \pm 7$ & $95 \pm 7$ & $77 \pm 8$ & $83 \pm 7$ & $93 \pm 4$ & $83 \pm 8$ & $86 \pm 8$ & $82 \pm 5$ \\
\hline 6-OH-BDE47 & $90 \pm 5$ & $92 \pm 5$ & $94 \pm 7$ & $79 \pm 11$ & $87 \pm 5$ & $84 \pm 5$ & $83 \pm 11$ & $93 \pm 6$ & $90 \pm 8$ \\
\hline 2'-OH-BDE68 & $81 \pm 12$ & $87 \pm 11$ & $94 \pm 6$ & $84 \pm 8$ & $86 \pm 8$ & $94 \pm 5$ & $73 \pm 8$ & $90 \pm 7$ & $93 \pm 9$ \\
\hline 6-OH-BDE85 & $83 \pm 7$ & $89 \pm 10$ & $88 \pm 6$ & $80 \pm 13$ & $82 \pm 9$ & $85 \pm 8$ & $82 \pm 9$ & $85 \pm 10$ & $88 \pm 12$ \\
\hline 5'-OH-BDE99 & $77 \pm 9$ & $84 \pm 5$ & $94 \pm 4$ & $82 \pm 14$ & $85 \pm 8$ & $92 \pm 8$ & $82 \pm 7$ & $87 \pm 13$ & $89 \pm 9$ \\
\hline 6'-OH-BDE99 & $85 \pm 13$ & $87 \pm 8$ & $95 \pm 3$ & $80 \pm 9$ & $86 \pm 7$ & $94 \pm 6$ & $74 \pm 11$ & $82 \pm 6$ & $86 \pm 6$ \\
\hline
\end{tabular}


Table 5

Spike recoveries and standard deviation ( $\% \pm \mathrm{SD}, n=3$ ) at $0.3,2$ and 5 ng for each target analyte using three different environmental matrices (plant, mollusk, fish).

\begin{tabular}{|c|c|c|c|c|c|c|c|c|c|}
\hline \multirow[t]{2}{*}{ Compounds } & \multicolumn{3}{|l|}{$\begin{array}{l}\text { Plant } \\
\% R \pm \mathrm{SD}\end{array}$} & \multicolumn{3}{|l|}{$\begin{array}{l}\text { Mollusk } \\
\% R \pm \mathrm{SD}\end{array}$} & \multicolumn{3}{|l|}{$\begin{array}{l}\text { Fish } \\
\% R \pm \mathrm{SD}\end{array}$} \\
\hline & $3 \mathrm{ng} / \mathrm{mL}$ & $20 \mathrm{ng} / \mathrm{mL}$ & $50 \mathrm{ng} / \mathrm{mL}$ & $3 \mathrm{ng} / \mathrm{mL}$ & $20 \mathrm{ng} / \mathrm{mL}$ & $50 \mathrm{ng} / \mathrm{mL}$ & $3 \mathrm{ng} / \mathrm{mL}$ & $20 \mathrm{ng} / \mathrm{mL}$ & $50 \mathrm{ng} / \mathrm{mL}$ \\
\hline BDE-28 & $84 \pm 7$ & $84 \pm 6$ & $89 \pm 9$ & $85 \pm 8$ & $86 \pm 7$ & $93 \pm 2$ & $83 \pm 7$ & $84 \pm 7$ & $86 \pm 8$ \\
\hline BDE-47 & $83 \pm 10$ & $91 \pm 5$ & $93 \pm 5$ & $74 \pm 8$ & $86 \pm 8$ & $89 \pm 6$ & $77 \pm 6$ & $91 \pm 8$ & $93 \pm 3$ \\
\hline BDE-66 & $79 \pm 8$ & $87 \pm 8$ & $91 \pm 5$ & $87 \pm 7$ & $96 \pm 5$ & $92 \pm 4$ & $85 \pm 9$ & $88 \pm 5$ & $94 \pm 4$ \\
\hline BDE-68 & $75 \pm 10$ & $96 \pm 7$ & $92 \pm 6$ & $75 \pm 5$ & $109 \pm 7$ & $95 \pm 6$ & $90 \pm 8$ & $91 \pm 8$ & $96 \pm 7$ \\
\hline BDE-85 & $83 \pm 6$ & $104 \pm 6$ & $85 \pm 7$ & $83 \pm 6$ & $85 \pm 9$ & $92 \pm 7$ & $81 \pm 5$ & $86 \pm 6$ & $89 \pm 6$ \\
\hline BDE-99 & $82 \pm 5$ & $94 \pm 5$ & $93 \pm 9$ & $77 \pm 6$ & $90 \pm 9$ & $92 \pm 11$ & $73 \pm 9$ & $88 \pm 7$ & $84 \pm 4$ \\
\hline BDE-138 & $79 \pm 9$ & $91 \pm 7$ & $94 \pm 4$ & $84 \pm 7$ & $88 \pm 5$ & $93 \pm 5$ & $83 \pm 6$ & $90 \pm 8$ & $92 \pm 6$ \\
\hline BDE-153 & $84 \pm 8$ & $84 \pm 6$ & $92 \pm 5$ & $86 \pm 9$ & $88 \pm 8$ & $85 \pm 6$ & $110 \pm 8$ & $86 \pm 8$ & $89 \pm 13$ \\
\hline BDE-154 & $92 \pm 6$ & $85 \pm 5$ & $91 \pm 7$ & $83 \pm 6$ & $92 \pm 6$ & $93 \pm 8$ & $78 \pm 9$ & $92 \pm 6$ & $95 \pm 6$ \\
\hline BDE-183 & $82 \pm 6$ & $92 \pm 8$ & $95 \pm 2$ & $84 \pm 12$ & $89 \pm 9$ & $87 \pm 6$ & $82 \pm 10$ & $88 \pm 9$ & $85 \pm 7$ \\
\hline 2'-MeO-BDE68 & $76 \pm 9$ & $90 \pm 6$ & $94 \pm 8$ & $76 \pm 6$ & $91 \pm 8$ & $95 \pm 9$ & $73 \pm 7$ & $84 \pm 5$ & $91 \pm 5$ \\
\hline 6-MeO-BDE47 & $87 \pm 8$ & $86 \pm 7$ & $93 \pm 7$ & $83 \pm 10$ & $95 \pm 11$ & $92 \pm 6$ & $91 \pm 8$ & $96 \pm 8$ & $94 \pm 4$ \\
\hline 3-MeO-BDE47 & $79 \pm 8$ & $88 \pm 8$ & $98 \pm 5$ & $103 \pm 5$ & $92 \pm 5$ & $95 \pm 4$ & $86 \pm 6$ & $90 \pm 7$ & $93 \pm 5$ \\
\hline 5-MeO-BDE47 & $74 \pm 7$ & $104 \pm 6$ & $94 \pm 3$ & $84 \pm 8$ & $85 \pm 7$ & $89 \pm 2$ & $83 \pm 7$ & $85 \pm 6$ & $89 \pm 4$ \\
\hline 4'-MeO-BDE49 & $87 \pm 7$ & $83 \pm 4$ & $93 \pm 6$ & $76 \pm 7$ & $91 \pm 6$ & $95 \pm 5$ & $78 \pm 9$ & $91 \pm 15$ & $95 \pm 3$ \\
\hline 4-MeO-BDE42 & $90 \pm 6$ & $91 \pm 5$ & $94 \pm 5$ & $81 \pm 7$ & $88 \pm 5$ & $85 \pm 4$ & $82 \pm 8$ & $90 \pm 8$ & $92 \pm 12$ \\
\hline 6'-MeO-BDE99 & $73 \pm 12$ & $90 \pm 10$ & $95 \pm 2$ & $84 \pm 6$ & $91 \pm 6$ & $94 \pm 6$ & $84 \pm 12$ & $92 \pm 7$ & $96 \pm 6$ \\
\hline 5'-MeO-BDE99 & $81 \pm 7$ & $90 \pm 8$ & $90 \pm 8$ & $83 \pm 9$ & $93 \pm 8$ & $95 \pm 7$ & $76 \pm 9$ & $107 \pm 6$ & $96 \pm 5$ \\
\hline 6-MeO-BDE85 & $90 \pm 5$ & $96 \pm 5$ & $93 \pm 7$ & $85 \pm 6$ & $92 \pm 6$ & $94 \pm 3$ & $74 \pm 5$ & $94 \pm 9$ & $91 \pm 8$ \\
\hline 3'-OH-BDE28 & $78 \pm 4$ & $87 \pm 8$ & $83 \pm 4$ & $82 \pm 8$ & $86 \pm 8$ & $83 \pm 12$ & $83 \pm 14$ & $87 \pm 7$ & $87 \pm 7$ \\
\hline 4-OH-BDE42 & $89 \pm 8$ & $86 \pm 8$ & $86 \pm 11$ & $77 \pm 8$ & $87 \pm 14$ & $79 \pm 5$ & $76 \pm 5$ & $89 \pm 4$ & $76 \pm 6$ \\
\hline 3-OH-BDE47 & $90 \pm 9$ & $88 \pm 13$ & $85 \pm 6$ & $79 \pm 5$ & $88 \pm 8$ & $95 \pm 11$ & $79 \pm 8$ & $91 \pm 6$ & $97 \pm 4$ \\
\hline $4^{\prime}-\mathrm{OH}-\mathrm{BDE} 49$ & $83 \pm 6$ & $93 \pm 8$ & $94 \pm 4$ & $84 \pm 7$ & $91 \pm 6$ & $94 \pm 2$ & $78 \pm 7$ & $87 \pm 7$ & $81 \pm 4$ \\
\hline 5-OH-BDE47 & $82 \pm 6$ & $85 \pm 11$ & $87 \pm 13$ & $83 \pm 13$ & $86 \pm 7$ & $83 \pm 6$ & $76 \pm 11$ & $88 \pm 9$ & $85 \pm 6$ \\
\hline 6-OH-BDE47 & $75 \pm 7$ & $92 \pm 5$ & $96 \pm 5$ & $81 \pm 8$ & $87 \pm 11$ & $82 \pm 7$ & $85 \pm 7$ & $96 \pm 8$ & $92 \pm 5$ \\
\hline 2'-OH-BDE68 & $81 \pm 5$ & $91 \pm 14$ & $93 \pm 4$ & $77 \pm 7$ & $85 \pm 4$ & $83 \pm 14$ & $77 \pm 6$ & $91 \pm 6$ & $95 \pm 7$ \\
\hline 6-OH-BDE85 & $79 \pm 14$ & $89 \pm 6$ & $81 \pm 5$ & $83 \pm 8$ & $87 \pm 12$ & $85 \pm 10$ & $82 \pm 9$ & $85 \pm 8$ & $88 \pm 7$ \\
\hline 5'-OH-BDE99 & $85 \pm 6$ & $87 \pm 7$ & $88 \pm 14$ & $75 \pm 7$ & $87 \pm 5$ & $83 \pm 8$ & $79 \pm 7$ & $86 \pm 11$ & $81 \pm 8$ \\
\hline 6'-OH-BDE99 & $81 \pm 8$ & $86 \pm 10$ & $95 \pm 6$ & $81 \pm 6$ & $85 \pm 7$ & $89 \pm 8$ & $84 \pm 13$ & $89 \pm 7$ & $86 \pm 6$ \\
\hline
\end{tabular}

Lupton et al. [33] proposed an LC-MS/MS method and the separation of nine $\mathrm{OH}-\mathrm{PBDEs}$ achieved in $35 \mathrm{~min}$. In another paper [34], eight OH-PBDEs can be separated by using a ternary mixture of $5 \mathrm{mM}$ ammonium acetate, acetonitrile and methanol as the mobile phases. Chang et al. [35] used acetonitrile and $0.1 \%$ formic acid in water as the mobile phases for the separation of ten $\mathrm{OH}-\mathrm{PBDEs}$ after derivatization with dansyl chloride. In our developed method, ten OH-PBDEs were separated completely in only 20 min without derivatization and no buffer was used in the mobile phase.

The separation of isomer on LC chromatographic column was known to be difficult. In the published work, only 2-4 $\mathrm{OH}-$ tetraBDE isomer were analyzed and the separating degree was not satisfied $[29,33,34]$. While using the improved methods in this work, six most frequently detected $\mathrm{OH}$-tetraBDE isomer, including two ortho-hydroxylated PBDEs (6-OH-BDE47 and 2'-OH-BDE68), two para-hydroxylated PBDEs (4'-OH-BDE49 and 4-OH-BDE42), two meta-hydroxylated PBDEs (3-OH-BDE47 and 5-OH-BDE47) [36], can be simultaneously identified, which were shown in Fig. 2.

MS/MS parameters were optimized by automatic procedures. In MS2 scan mode, the $[\mathrm{M}-\mathrm{H}]^{-}$base peak formed at $m / z 578.6$ for $\mathrm{OH}-$ pentaBDE, $m / z 500.7$ for OH-tetraBDE and $m / z 420.7$ for OH-triBDE. Following the successful detection of the $[\mathrm{M}-\mathrm{H}]^{-}$ion, fragmentor voltage was optimized to obtain the most intense signal. Subsequently, product ion scan mode was used to choose product ions and optimize collision energy. The typical fragment ions of analytes were consistent with the results in other papers [29,33,34]. The formation of specific fragment ions allowed the efficient analytical method based on the MRM mode. Two specific MRM transitions were used for each compound, one for qualitative analysis and another for quantification. The $[\mathrm{M}-\mathrm{H}]^{-}$precursor ion, the product ions, the chosen MRM transitions with optimized fragmentor (frag) and collision energy (CE) for each OH-PBDEs are summarized in Table 2.

\subsection{Performance of the method}

To evaluate the performance of the developed method, linearity, recovery, precision, repeatability and sensitivity were examined (Tables 3-5). Good linearity was obtained with $r^{2}$ over 0.992 for PBDEs, 0.995 for MeO-PBDEs and 0.992 for OH-PBDEs under a concentration range from 2 to $200 \mathrm{ng} / \mathrm{mL}$. The equipment detection limits (EDLs) ranged from 0.24 to $0.75 \mathrm{ng} / \mathrm{mL}$ for PBDEs and $\mathrm{MeO}$-analytes and from 0.01 to $0.10 \mathrm{ng} / \mathrm{mL}$ for $\mathrm{OH}-\mathrm{PBDEs}$. According to the work of Lacorte et al. [37], blank samples were used to evaluate the performance of the optimized approach because there is no reference material available presently for $\mathrm{MeO}$ - and OH-PBDEs. For OH-PBDEs, MDLs were $3.2-11.6 \mathrm{pg} / \mathrm{L}$ in water sample and $2.8-18.4 \mathrm{pg} / \mathrm{g}$ dry weight $(\mathrm{dw})$ in solid samples. For PBDEs and MeO-PBDEs, MDLs were $48.8-150.3 \mathrm{pg} / \mathrm{L}$ in water sample and $46.5-170.8 \mathrm{pg} / \mathrm{g}$ dry weight in solid samples. The MDLs of hydroxylated analytes determined by LC-MS/MS were over one order of magnitude lower than other reported non-derivatization LC-MS/MS methods [29,34], and similar to the results for OH-PBDEs after derivatization with dansyl chloride by LC-MS/MS method [35]. This high sensitivity could be very helpful in detecting trace OH-PBDEs in aquatic system and for studying the metabolic mechanism in organisms [36]. Recoveries for all the analytes in six different environmental matrices were assessed by spiking with each compound. The mean $(n=3)$ recoveries for 29 target analytes in all the matrices ranged from $71 \%$ to $113 \%$. Repeatability was evaluated by intra- and inter-assay variation. At the spiking level of $5 \mathrm{ng}$, repeatability, as measured by relative standard deviation (\%RSD), was between $4-12 \%$ for all analytes. The precision of the method, obtained as the relative standard deviations (RSDs) of analyte recoveries, were $2-16 \%$ in all the spiking experiments. Because of absence of certified reference materials (CRMs), reported method was used [37] for the accuracy assessment. The results of Pseudosciaena polyactis sample showed that the detected concentrations by our method $(1.5 \mathrm{ng} / \mathrm{g} \mathrm{dw}$ for $6-\mathrm{MeO}-\mathrm{BDE}-47)$ close to that by 
Table 6

Concentrations (ng/g dry weight) of MeO-PBDEs detected in marine animals obtained from the Bohai Sea and the Donghai Sea, China.

\begin{tabular}{|c|c|c|c|c|}
\hline Sampling site & Tianjin & Tianjin & Weihai & Taizhou \\
\hline Sample name & Mya arenaria & Mytilus edulis & Mytilus edulis & Pseudosciaena polyactis \\
\hline 2'-MeO-BDE-68 & n.d. ${ }^{\mathrm{a}}$ & 2.1 & 1.0 & n.d. \\
\hline 6-MeO-BDE-47 & n.d. & 1.4 & 1.3 & 1.5 \\
\hline 3-MeO-BDE-47 & n.d. & n.d. & n.d. & n.d. \\
\hline 5-MeO-BDE-47 & 2.0 & 3.2 & n.d. & n.d. \\
\hline $4^{\prime}-\mathrm{MeO}-\mathrm{BDE}-49$ & n.d. & n.d. & n.d. & n.d. \\
\hline 4-MeO-BDE-42 & n.d. & n.d. & n.d. & n.d. \\
\hline 6'-MeO-BDE-99 & n.d. & n.d. & n.d. & n.d. \\
\hline 5'-MeO-BDE-99 & n.d. & n.d. & n.d. & n.d. \\
\hline 6-MeO-BDE-85 & n.d. & n.d. & n.d. & n.d. \\
\hline
\end{tabular}

a None-detectable.

reported method $(1.7 \mathrm{ng} / \mathrm{g} \mathrm{dw}$ for 6-MeO-BDE-47). Therefore, the method is reliable and the linearity, recovery, repeatability, and sensitivity of the method were demonstrated to be suitable for analyzing PBDEs, MeO-PBDEs, and OH-PBDEs.

\subsection{Application of the method to environmental samples}

Using the developed method, OH-PBDEs and MeO-PBDEs in mollusk and fish samples were studied. Three MeO-PBDEs: 5MeO-BDE-47, 6-MeO-BDE-47 and 2'-MeO-BDE-68 were detected in Mytilus edulis, Mya arenaria and Pseudosciaena polyactis samples (Table 6), in which 6-MeO-BDE-47 and 2'-MeO-BDE-68 were frequently determined in wildlife $[2,5,10,38]$. The concentration of $\mathrm{MeO}-\mathrm{PBDEs}$ in Mytilus edulis collected from Tianjin ranged from 1.4 to $3.2 \mathrm{ng} / \mathrm{g}$ dry weight. Mytilus edulis collected from Weihai presented a lower concentration than that from Tianjin, with $1.0 \mathrm{ng} / \mathrm{g}$ dry weight for 2'-MeO-BDE-68, $1.3 \mathrm{ng} / \mathrm{g}$ dry weight for 6-MeO-BDE47. 5-MeO-BDE-47 was only detected in Mya arenaria collected from Tianjin with a concentration of $2.0 \mathrm{ng} / \mathrm{g}$ dry weight and in Pseudosciaena polyactis samples collected from Taizhou, 6-MeOBDE-47 (1.5 ng/g dry weight) was found. The different distributions of MeO-PBDE congeners in different species might be related to the different sampling location and metabolism [35]. The concentrations were comparable to the results of Mytilus edulis ( $8.8 \mathrm{ng} / \mathrm{g}$ lipid weight for $6-\mathrm{MeO}-\mathrm{BDE}-47$ and $2.8 \mathrm{ng} / \mathrm{g}$ lipid weight for $2^{\prime}$-MeOBDE-68) collected from the Hudson Bay region of northeastern Canada [10]. All the analyzed OH-PBDEs were below the detection limits, which were also reported to be much lower than that of MeO-PBDEs in the marine food web [39].

\section{Conclusions}

In the present study, a reliable, robust, efficient, and sensitive analytical method was developed for the identification and quantification of 10 PBDEs, 19 potential metabolized MeO-PBDEs and $\mathrm{OH}-\mathrm{PBDEs}$ in six environmental matrices including abiotic and biotic samples. A water-impregnated silica separating column was found to be most suitable to be used for the simultaneous separation of PBDEs, MeO-PBDEs and OH-PBDEs. The linearity, recovery and repeatability of the method were demonstrated in acceptable ranges. The selectivity and sensitivity of LC-MS/MS methods were further improved comparing with reported methods and made it more efficient in OH-PBDEs analysis.

\section{Acknowledgements}

This work was jointly supported by the National Basic Research Program of China (No. 2009CB421605), the National Natural Science Foundation of China (Nos. 20877080 and 20907062), and National Key Water Program (No. 2009ZX07527-005).

\section{References}

[1] D. Ueno, C. Darling, M. Alaee, G. Pacepavicius, C. Teixeira, L. Campbell, R.J. Letcher, A. Bergman, G. Marsh, D. Muir, Environ. Sci. Technol. 42 (2008) 1657-1664.

[2] A. Malmvarn, G. Marsh, L. Kautsky, M. Athanasiadou, A. Bergman, L. Asplund, Environ. Sci. Technol. 39 (2005) 2990-2997.

[3] J. Verreault, G.V. Gabrielsen, S.G. Chu, D.C.G. Muir, M. Andersen, A. Hamaed, R.J. Letcher, Environ. Sci. Technol. 39 (2005) 6021-6028.

[4] P.S. Haglund, D.R. Zook, H.R. Buser, J.W. Hu, Environ. Sci. Technol. 31 (1997) 3281-3287.

[5] G. Marsh, M. Athanasiadou, A. Bergman, L. Asplund, Environ. Sci. Technol. 38 (2004) 10-18.

[6] S. Sinkkonen, A.L. Rantalainen, J. Paasivirta, M. Lahtipera, Chemosphere 56 (2004) 767-775.

[7] K. Valters, H.X. Li, M. Alaee, I. D’Sa, G. Marsh, A. Bergman, R.J. Letcher, Environ. Sci. Technol. 39 (2005) 5612-5619.

[8] L. Hovander, T. Malmberg, M. Athanasiadou, L. Athanassiadis, S. Rahm, A. Bergman, E.K. Wehler, Arch. Environ. Contam. Toxicol. 42 (2002) 105-117.

[9] S. Lacorte, M.G. Ikonomou, Chemosphere 74 (2009) 412-420.

[10] B.C. Kelly, M.G. Ikonomou, J.D. Blair, F.A.P.C. Gobas, Environ. Sci. Technol. 42 (2008) 7069-7077.

[11] I.A.T.M. Meerts, R.J. Letcher, S. Hoving, G. Marsh, A. Bergman, J.G. Lemmen, B. van der Burg, A. Brouwer, Environ. Health Perspect. 109 (2001) 399-407.

[12] M. Mercado-Feliciano, R.M. Bigsby, Environ. Health Perspect. 116 (2008) 1315-1321.

[13] R.F. Canton, D.E.A. Scholten, G. Marsh, P.C. De Jong, M. Van den Berg, Toxicol. Appl. Pharmacol. 227 (2008) 68-75.

[14] M. Harju, T. Hamers, J.H. Kamstra, E. Sonneveld, J.P. Boon, M. Tysklind, P.L. Andersson, Environ. Toxicol. Chem. 26 (2007) 816-826.

[15] D. Handayani, R.A. Edrada, P. Proksch, V. Wray, L. Witte, R.W.M. Van Soest, A. Kunzmann, Soedarsono, J. Nat. Prod. 60 (1997) 1313-1316.

[16] E.L. Teuten, L. Xu, C.M. Reddy, Science 308 (2005) 1413.

[17] X. Fu, M.B. Hossain, F.J. Schmitz, D. vanderHelm, J. Org. Chem. 62 (1997) 3810-3819.

[18] J. Salva, D.J. Faulkner, J. Nat. Prod. 53 (1990) 757-760.

[19] T. Malmberg, M. Athanasiadou, G. Marsh, I. Brandt, A. Bergmant, Environ. Sci. Technol. 39 (2005) 5342-5348.

[20] A. Morck, H. Hakk, U. Orn, E.K. Wehler, Drug Metab. Dispos. 31 (2003) 900907.

[21] G. Marsh, M. Athanasiadou, I. Athanassiadis, A. Sandholm, Chemosphere 63 (2006) 690-697.

[22] H. Hakk, G. Larsen, E. Klasson-Wehler, Xenobiotica 32 (2002) 369-382.

[23] H. Hakk, J. Huwe, M. Low, D. Rutherford, G. Larsen, Xenobiotica 36 (2006) 79-94.

[24] Y. Wan, S. Wiseman, H. Chang, X.W. Zhang, P.D. Jones, M. Hecker, K. Kannan, S. Tanabe, J.Y. Hu, M.H.W. Lam, J.P. Giesy, Environ. Sci. Technol. 43 (2009) 7536-7542.

[25] A. Malmvarn, Y. Zebuhr, L. Kautsky, A. Bergman, L. Asplund, Chemosphere 72 (2008) 910-916.

[26] H. Routti, R.J. Letcher, S.G. Chu, B. Van Bavel, G.W. Gabrielsen, Environ. Sci. Technol. 43 (2009) 3494-3499.

[27] J.H. Yen, W.C. Liao, W.C. Chen, Y.S. Wang, J. Hazard. Mater. 165 (2009) 518524.

[28] E. Rogers, M. Petreas, J.S. Park, G.M. Zhao, M.J. Charles, J. Chromatogr. B 813 (2004) 269-285.

[29] Y. Kato, S. Okada, K. Atobe, T. Endo, F. Matsubara, T. Oguma, K. Haraguchi, Anal. Chem. 81 (2009) 5942-5948.

[30] K. Haraguchi, Y. Kato, K. Atobe, S. Okada, T. Endo, F. Matsubara, T. Oguma, Anal. Chem. 80 (2008) 9748-9755.

[31] L. Debrauwer, A. Riu, M. Jouahri, E. Rathahao, I. Jouanin, J.P. Antignac, R. Cariou, B. Le Bizec, D. Zalko, J. Chromatogr. A 1082 (2005) 98-109.

[32] H.X. Li, K.G. Drouillard, E. Bennett, G.D. Haffner, R.J. Letcher, Environ. Sci. Technol. 37 (2003) 832-839.

[33] S.J. Lupton, B.P. McGarrigle, J.R. Olson, T.D. Wood, D.S. Aga, Rapid Commun. Mass Spectrom. 24 (2010) 2227-2235. 
[34] S. Mas, O. Jauregui, F. Rubio, A. de Juan, R. Tauler, S. Lacorte, J. Mass Spectrom. 42 (2007) 890-899.

[35] H. Chang, Y. Wan, J. Naile, X.W. Zhang, S. Wiseman, M. Hecker, M.H.W. Lam, J.P. Giesy, P.D. Jones, J. Chromatogr. A 1217 (2010) 506-513.

[36] S.B. Wiseman, Y. Wan, H. Chang, X.W. Zhang, M. Hecker, P.D. Jones, J.P. Giesy, Mar. Pollut. Bull. 63 (2011) 179-188.
[37] S. Lacorte, M.G. Ikonomou, M. Fischer, J. Chromatogr. A 1217 (2010) 337-347.

[38] H.S. Wang, J. Du, K.L. Ho, H.M. Leung, M.H.W. Lam, J.P. Giesy, C.K.C. Wong, M.H Wong, J. Hazard. Mater. 192 (2011) 374-380.

[39] U. Orn, E. Klasson-Wehler, Xenobiotica 28 (1998) 199-211. 